\title{
A Stock Market Trading System using Deep Neural Network
}

\author{
Bang Xiang Yong, Mohd Rozaini Abdul Rahim, Ahmad Shahidan Abdullah \\ Faculty of Electrical Engineering, Universiti Teknologi Malaysia, \\ 81310 Skudai, Johor, Malaysia \\ yongbangxiang@gmail.com,mrozaini.ar@gmail.com, ashahidan@utm.my
}

\begin{abstract}
The stock market prediction is a lucrative field of interest with promising profit and covered with landmines for the unprecedented. The markets are complex, non-linear and chaotic in nature which poses huge difficulties to predict the prices accurately. In this paper, a stock trading system utilizing feed-forward deep neural network (DNN) to forecast index price of Singapore stock market using the FTSE Straits Time Index (STI) in t days ahead is proposed and tested through market simulations on historical daily prices. There are 40 input nodes of DNN which are the past 10 days' opening, closing, minimum and maximum prices and consist of 3 hidden layers with 10 neurons per layer. The training algorithm used is stochastic gradient descent with back-propagation and is accelerated with multi-core processing. A trading system is proposed which utilizes the DNN forecasting results with defined entry and exit rules to enter a trade. DNN performance is evaluated using RMSE and MAPE. The overall trading system shows promising results with a profit factor of $18.67,70.83 \%$ profitable trades and Sharpe ratio of 5.34 based on market simulation on test data.
\end{abstract}

Keywords: deep learning, stock market prediction, trading system

\section{Introduction}

Stock market prediction remains a lucrative field of research with promising profits for investors and researchers. However, there are challenges in predicting the stock markets accurately and precisely as the markets are complex, non-linear and chaotic in nature which calls for more powerful methods to tackle this problem.

Many artificial intelligence methods have been employed to predict stock market prices. Artificial neural networks (ANN) remain a popular choice for this task and are widely studied [1] and have been shown to exhibit good performance [2].

More recently, deep learning has emerged an improved method over conventional neural networks for various applications such as recognition system, natural language processing and medical sciences and has shown astonishing results [3]. It has also been applied in financial markets for prediction of stock prices using textual news data and numerical data [4] [5]. Experiment has been conducted to test its profitability and performance as a trading system in the stock and commodity market [6]. 
However, there is still a lack of studies conducted on the usage of deep learning as an integral part of a trading system.

In this paper, we propose a trading system for stock market which utilizes the prediction of DNN to generate trading signals, and evaluate its performance in the stock market as an attempt to convert the DNN predictions into a profitable system.

\section{Methodology}

This section describes the data set, process of training and testing the deep neural network and trading rules applied on the prediction output to test the profitability of the trading system.

\subsection{Data Set}

The data set used throughout this study consists of historical data of the opening, closing, maximum and minimum prices of FTSE Straits Time Index (STI) which is deemed to be the barometer of Singapore stock market. The opening price is the price at the beginning of the day. The closing price is the price at the end of the day. As the price fluctuates throughout the day, there is a maximum and minimum price which is recorded. Each time series data of stock ranged from $1^{\text {st }}$ January 2010 until $3^{\text {rd }}$ January 2017 with a period of 1769 days where the market is open. They were obtained from Yahoo Finance. The dataset is split into train and test sets with 75-25 ratio whereby evaluations are conducted using the test sets.

\subsection{Deep Neural Network}

Deep neural network is a special type of artificial neural network characterized by its architecture which consists of higher number of hidden layers and neurons compared to conventional neural network. Higher number of hidden layers exhibits increased capability of high-level features extraction for every added hidden layer [7]. Hence, the raw input data is not required to be pre-processed using features extraction methods compared to conventional neural network.

The principle for forecasting is based on windowing method, that is to use the opening, closing, maximum and minimum prices of $n-1, n-2, \ldots n-10$ days as inputs to the DNN to output the predicted closing price of $t$ days ahead. The number of prior working days is selected based on [8] which has shown promising results. Multiple models need to be trained for every forecast of $t$ days ahead.

In this paper, the architecture of DNN comprises of an input layer with 40 input nodes, 3 hidden layers with the composition of 10-10-10 neurons respectively and an output layer with a single node which outputs the stock price in $t$ days ahead. The DNN architecture is depicted in Fig. 1. 


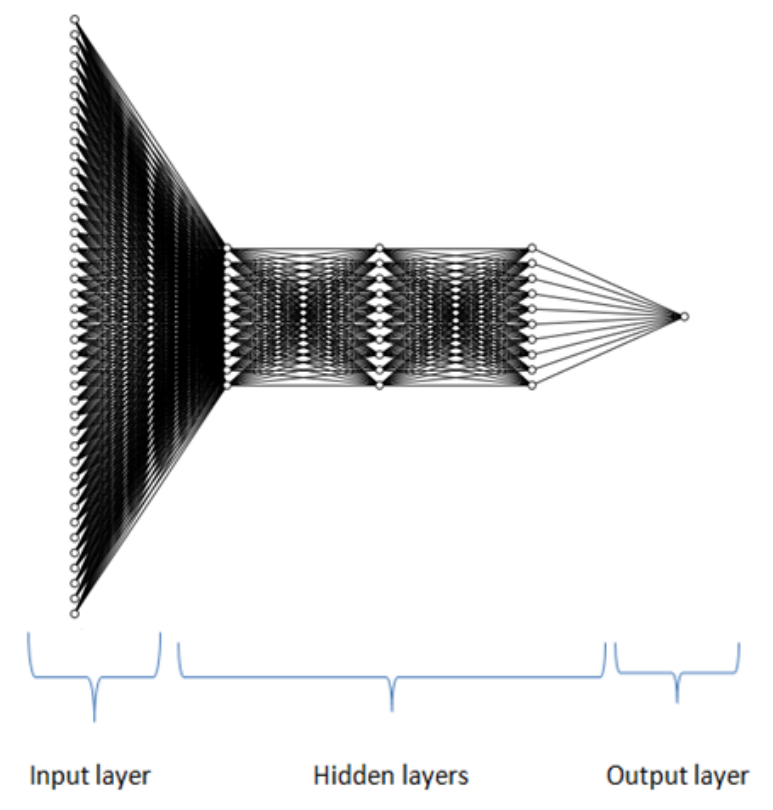

Fig. 1. Feed-forward deep neural network architecture used for prediction model of stock price of $n+t$ day

\subsection{Network Training}

Stochastic gradient descent and back-propagation is used as the learning algorithm. Training the DNN is an expensive process with 40 input variables and high number of neurons. To address this issue, multi-core processing method is used using Hogwild algorithm [9], which is a lock-free parallelization scheme whereby each core handles separate subsets of the training data. The result of training DNN is a forecast model which can be used to predict the closing price of $n+t$ day.

\subsection{Proposed Trading System}

The rules of a trading system tell the investor when to buy or sell the stocks which will result in a profitable trade. The proposed trading system uses the results of predicted closing stock prices of $n, n+1$ and $n+2$ days.

The proposed trading rules employ the logic of buying stocks when the forecasted closing price is higher than the current opening price, and selling all stocks (if any) in possession if the forecasted closing price is lower than current opening price. The 
effect of trading rules was investigated using different combination of $n+t$ day forecast. Let close $_{t}$ be the predicted closing price at $n+t$ day and open be the opening price of $\mathrm{n}$-th day. Trading rules used are divided into entry and exit rules. Different entry rules are proposed to be tested on the trading system to compare its performance. The trading rules used are as follows:

Entry rules:

1) Buy when close $_{0}>$ open

2) Buy when close $_{0}$ and close $_{1}>$ open

3) Buy when close $_{0}$ and close $_{1}$ and close $_{2}>$ open

4) Buy when close $_{0}$ and close $_{1}$ and close $_{2}$ and close $_{3}>$ open

5) Buy when close $_{0}$ and close $_{1}$ and close $_{2}$ and close $_{3}$ and close $_{4}>$ open

Exit Rule:

1) Sell all when close $_{0}>$ open

It is important to note that the proposed trading system do not consider the option of shorting stocks. For each buy signal, an equal amount of stock is bought. Also, the system allows stacking of bought stocks for consecutive buy signals, and sells all stocks in account for any sell signal. If there is no stock in hand when a sell signal is met, the system does nothing.

\section{Results}

The evaluation is conducted on two components namely the stock prediction model and performance of trading system. All evaluations are performed on the testing set.

\subsection{Evaluation of Stock Prediction Model}

The predicted closing price for the testing sets are plotted on the graph to visualize the actual versus the predicted price as depicted in Fig 2, 3, 4,5 and 6. 


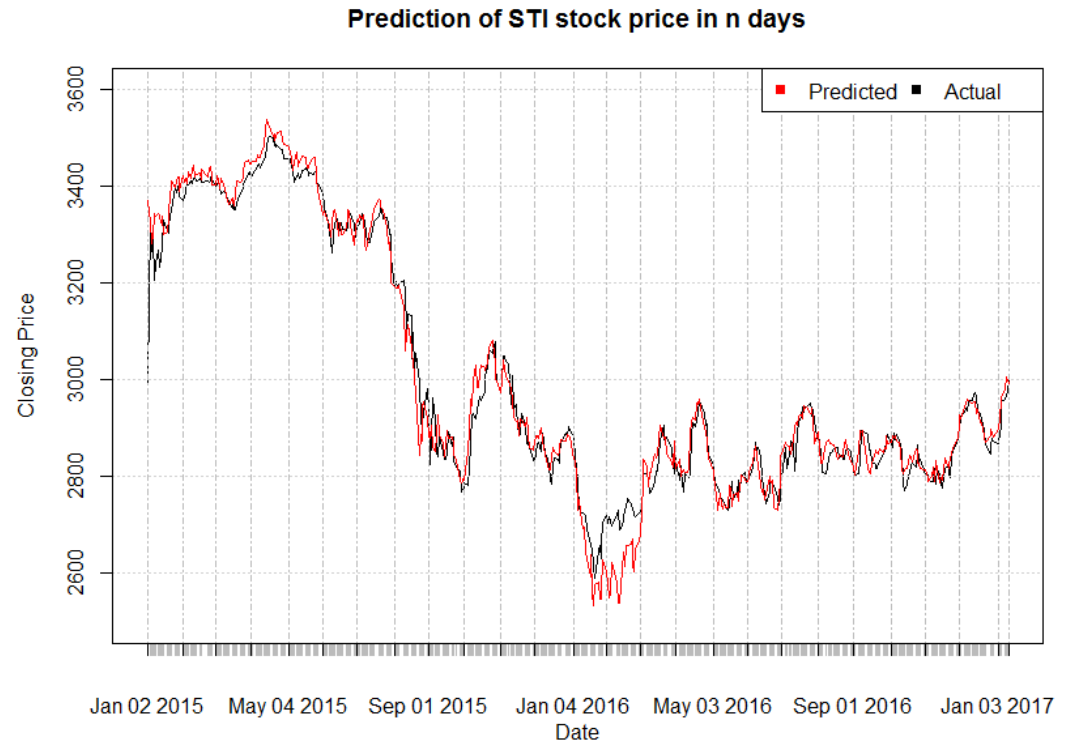

Fig. 2. Prediction of STI stock price in $\boldsymbol{n}$ days

Prediction of STI stock price in $n+1$ days

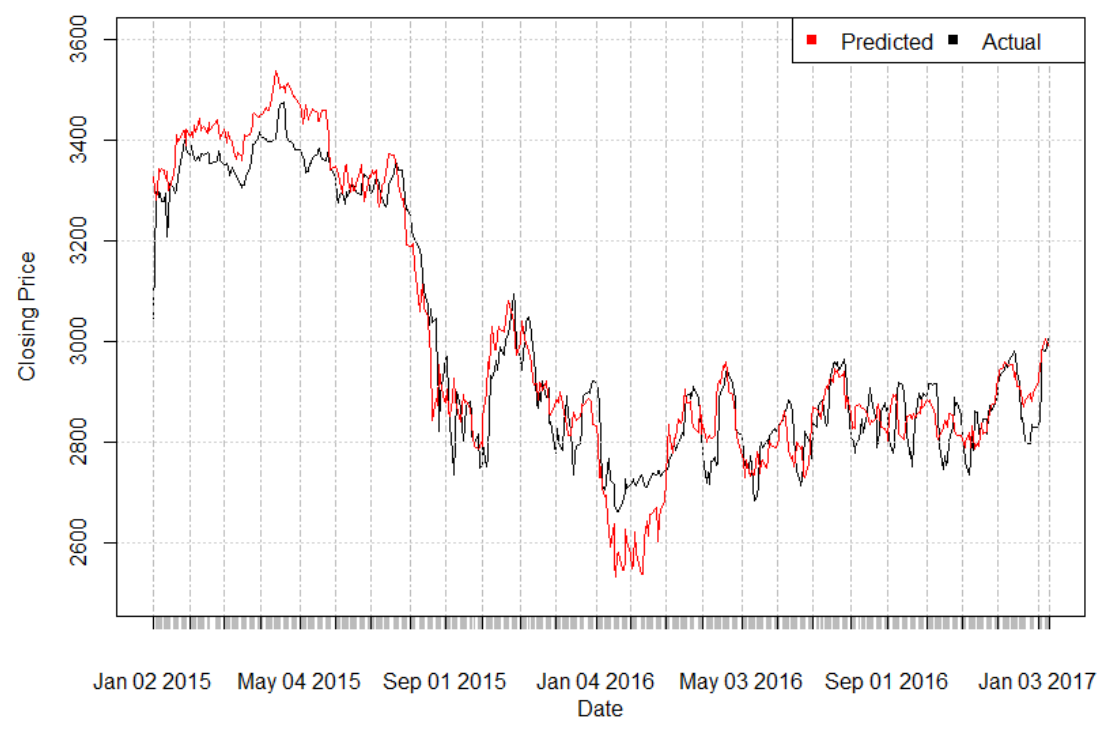

Fig. 3. Prediction of STI stock price in $n+1$ days 


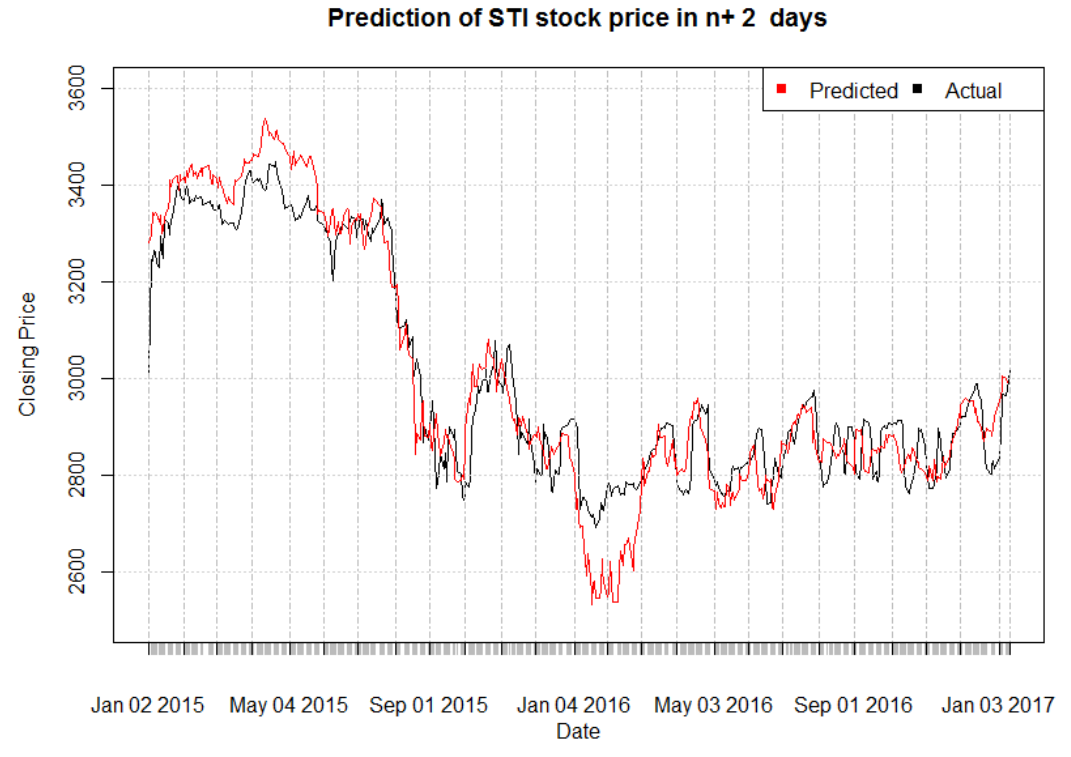

Fig. 4. Prediction of STI stock price in $n+2$ days

Prediction of STI stock price in $n+3$ days

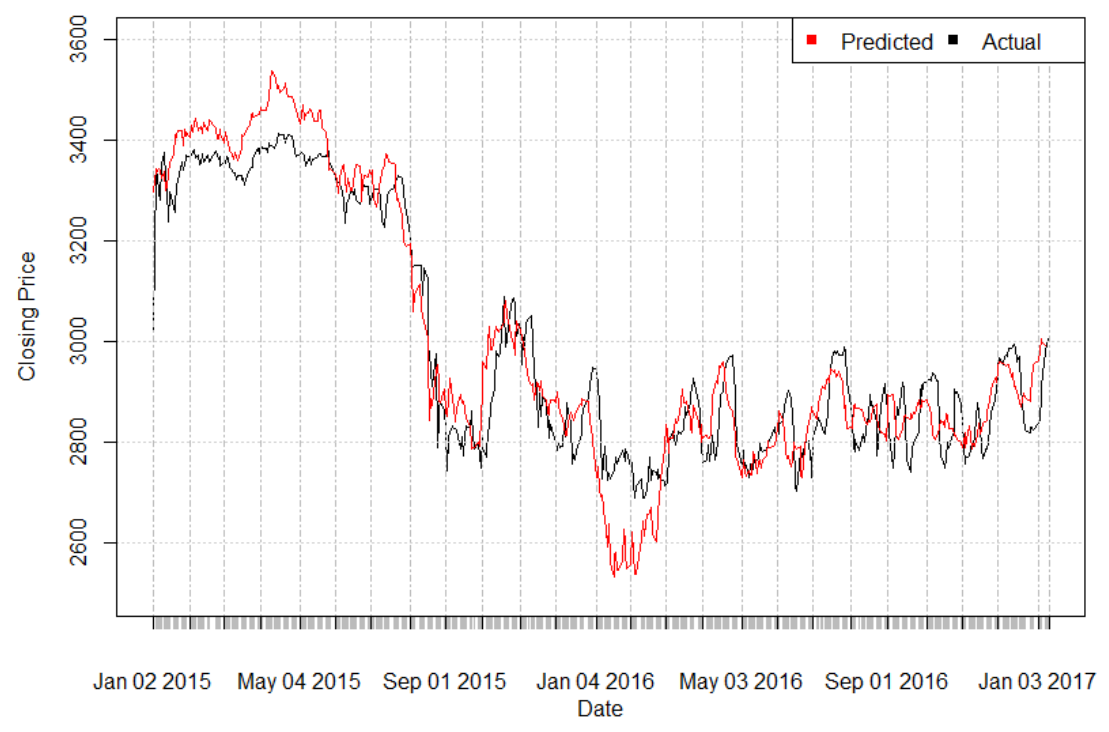

Fig. 5. Prediction of STI stock price in $n+3$ days 


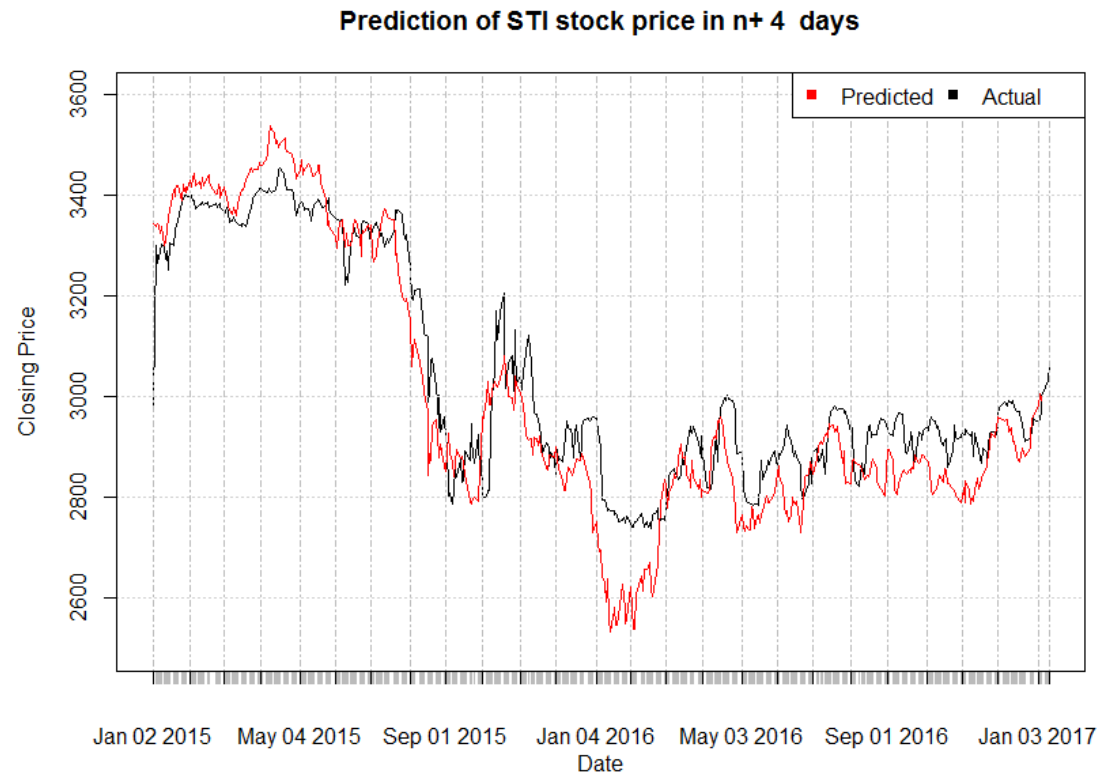

Fig. 6. Prediction of STI stock price in $n+4$ days

The evaluation metrics used for the prediction models are the conventional root mean square error (RMSE) and mean absolute percentage error (MAPE) which is a statistical measure of prediction accuracy of a forecast model. Different models are compared for closing price of $n, n+1, n+2, n+3, n+4$ days and are shown in Table 1 .

Table 1. RMSE of closing stock price prediction models of $t$ days ahead.

\begin{tabular}{lll}
\hline$t$ (days) & RMSE & MAPE \\
\hline 0 (Today) & 32.77 & 0.75 \\
1 & 48.40 & 1.20 \\
2 & 51.38 & 1.26 \\
3 & 72.00 & 1.83 \\
4 & 74.64 & 1.84 \\
\hline
\end{tabular}

As the number of days increases for the forecast, the generalization error increases as well. This shows that it is less accurate to predict the price further into the future compared to more recent forecasts. Note that since the model uses windowing method, in the beginning of the time series where the past stock prices are not available, the prediction result is not available as well. 


\subsection{Evaluation of Trading System}

The trading system is simulated on the test data using different entry rules as stated in the methodology. The metrics used are profit factor, Sharpe ratio and percentage of profitable trade and results are tabulated in Table 2.

Table 2. Performance of trading system using different entry rules

\begin{tabular}{lccc}
\hline Entry Rule & $\begin{array}{l}\text { Profit } \\
\text { Factor }\end{array}$ & $\begin{array}{l}\text { Profitable } \\
\text { Trades (\%) }\end{array}$ & $\begin{array}{l}\text { Sharpe } \\
\text { Ratio }\end{array}$ \\
\hline 1 & 2.54 & 47.37 & 2.13 \\
2 & 4.92 & 52.08 & 3.21 \\
3 & 14.32 & 67.74 & 4.69 \\
4 & 15.31 & 65.52 & 4.80 \\
5 & 18.67 & 70.83 & 5.34 \\
\hline
\end{tabular}

It is shown that the best performance is exhibited by using the entry rule 5 which enters a buy trade only when the forecasted closing prices of $n, n+1, n+2, \ldots n+4$ day are higher than the opening price of $n$-th day. This can be attributed to the higher chance of entering a profitable trade if there is an upward trend and ultimately increases the profit factor. This shows that the trading system which uses multiple steps of prediction from deep neural network can be profitable for the investors.

The market simulation of the transactions made using the trading system and its cumulative return curve is shown in Fig. 7 where the green triangle depicts a buy signal and the red triangle depicts a sell signal. 

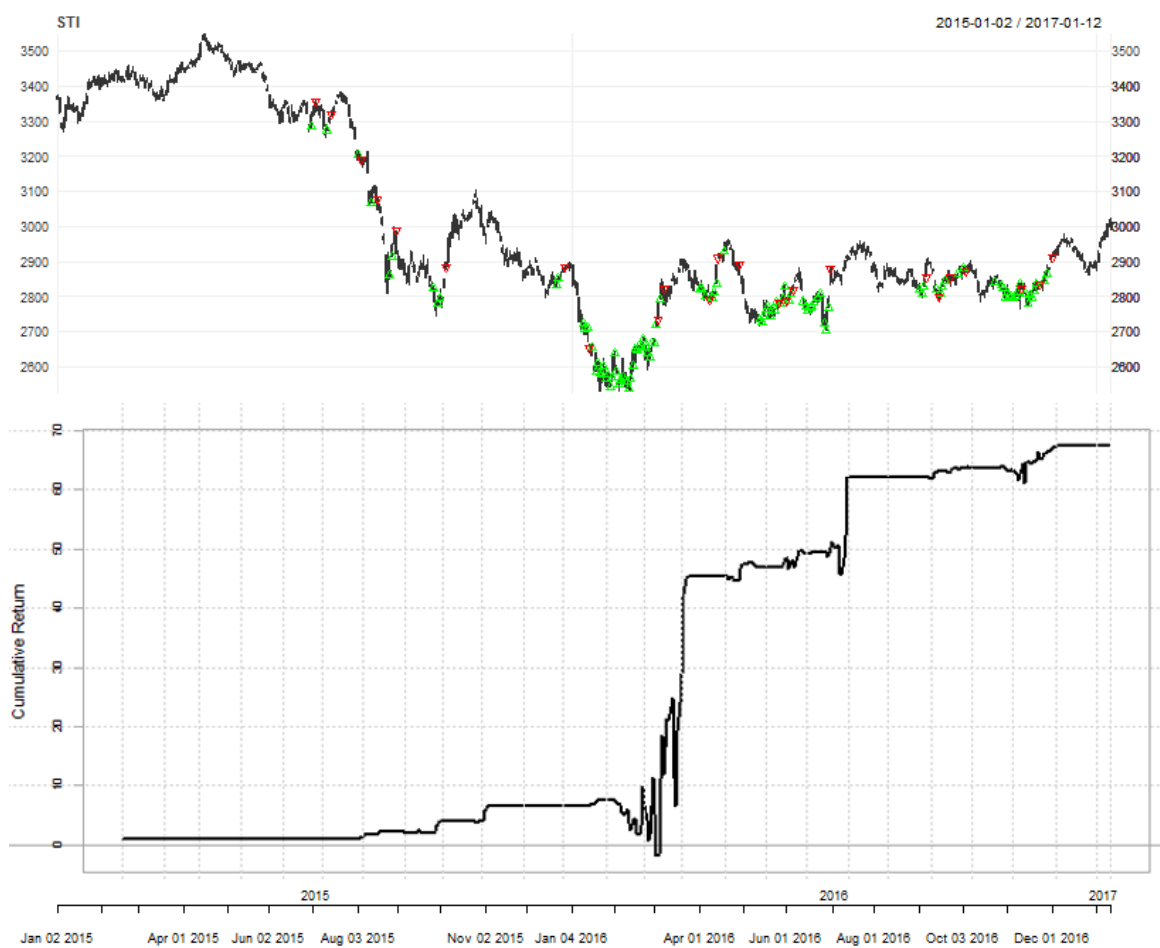

Fig. 7. Market simulation results of DNN trading system on test data

\section{Conclusion}

In this paper, a stock market trading system is proposed which uses deep neural network as part of its core components. The DNN uses historical data prices to forecast stock prices of $t$ days ahead which is incorporated in the trading system to make buy and sell decisions. The effect of varying the number of steps of the forecast model was investigated and it is shown that the forecast further into the future yields less accurate results. The consequences of varying the trading rules were also studied and the trading system with best performance was determined to have a profit factor of $18.67,70.83 \%$ profitable trades and Sharpe ratio of 5.34 .

In the future, different types of deep learning algorithm can be investigated such as Deep Boltzmann machine and Deep Q-networks. Better trading rules can also be investigated by using more advanced methods such as evolutionary programming. There is also potential for using different inputs of data such as correlated commodity and currency value, and varying the time frames such as every minute or hourly data. There are huge potential for the expandability of this trading system and its robustness and reliability should also be tested on other stock markets. 


\section{Acknowledgement}

The authors would like to thank all who contributed toward making this research successful. The authors wish to express their gratitude to Ministry of Higher Education (MOHE), Research Management Center (RMC) for the sponsorship, and Advanced Telecommunication Technology Research Group, Universiti Teknologi Malaysia for the financial support and advice for this project. (VOT number: Q.J130000.2623.12J82).

\section{References}

1. Vui, C.S., et al. A review of stock market prediction with Artificial neural network (ANN). in 2013 IEEE International Conference on Control System, Computing and Engineering. 2013.

2. Yetis, Y., H. Kaplan, and M. Jamshidi. Stock market prediction by using artificial neural network. in 2014 World Automation Congress (WAC). 2014.

3. Soniya, S. Paul, and L. Singh. A review on advances in deep learning. in 2015 IEEE Workshop on Computational Intelligence: Theories, Applications and Future Directions (WCI). 2015.

4. Akita, R., et al. Deep learning for stock prediction using numerical and textual information. in 2016 IEEE/ACIS 15th International Conference on Computer and Information Science (ICIS). 2016.

5. Day, M.Y. and C.C. Lee. Deep learning for financial sentiment analysis on finance news providers. in 2016 IEEE/ACM International Conference on Advances in Social Networks Analysis and Mining (ASONAM). 2016.

6. $\quad$ Deng, Y., et al., Deep Direct Reinforcement Learning for Financial Signal Representation and Trading. IEEE Transactions on Neural Networks and Learning Systems, 2016. PP(99): p. 1-12.

7. Sun, Y., X. Wang, and X. Tang. Deep learning face representation from predicting 10,000 classes. in Proceedings of the IEEE Conference on Computer Vision and Pattern Recognition. 2014.

8. Moghaddam, A.H., M.H. Moghaddam, and M. Esfandyari, Stock market index prediction using artificial neural network. Journal of Economics, Finance and Administrative Science, 2016. 21(41): p. 89-93.

9. Recht, B., et al. Hogwild: A lock-free approach to parallelizing stochastic gradient descent. in Advances in Neural Information Processing Systems. 2011. 